führen. Bis zum 31. Dezember 1969 sind bei der Kommission immerhin sieben Staatenbeschwerden und 4334 Individualbeschwerden eingegangen.

Diese neue Stufe in der Entwicklung des Individualrechtsschutzes im Völkerrecht und damit des Völkerrechts überhaupt verdient in jedem Falle Aufmerksamkeit. Die materielle Rechtsfortbildung hinsichtlich der Grund- und Menschenrechte ist aber zweitens auch deshalb für viele Leser dieser Zeitschrift von Bedeutung, weil zahlreiche Staaten der Dritten Welt in ihre Verfassungen oder sonstige Gesetzgebungsakte Menschenrechtskataloge (bills of rights) aufgenommen haben, die inhaltlich an dem Vorbild der Europäischen Menschenrechtskonvention orientiert sind (vgl. dazu den Aufsatz von J. Read in diesem Heft, S. 21-47). Die materielle Anwendung der Konvention, die Interpretation der einzelnen Menschenrechte (unabhängig von den besonderen Verfahrensmodalitäten des europäischen Systems) wird sie deshalb für ihre eigene Rechtspraxis interessieren, ohne daß damit in irgendeiner Weise für eine kritiklose Rezeption plädiert wird. Für die hier entstehenden Schwierigkeiten und Probleme sei nochmals auf den Aufsatz von J. Read in diesem Heft verwiesen.

Im einzelnen ist der angezeigte Band weder Analyse noch Monographie, sondern ein Hilfsmittel, Nachschlagewerk, das in erster Linie die Benutzung der ersten zehn Bände der europäischen Menschenrechtsjudikatur im Annuaire de la Convention Européenne des Droits de l'Homme erleichtern soll. Er enthält zunächst eine tabellarische Übersicht über alle im Berichtszeitraum sachlich behandelten Beschwerden mit Angaben über Art und Datum der sie erledigenden Entscheidung mit den zugehörigen Fundstellen im Annuaire. Daran schließen sich im Hauptteil Auszüge aus oder Zusammenfassungen von Entscheidungen der Kommission oder des Gerichtshofes an. Sie sind nach allgemeinen Grundsätzen, im übrigen nach den fortlaufenden Artikeln der Konvention geordnet. Dabei wird sofort der sachliche Schwerpunkt der Schutzverfahren nach der Konvention deutlich. Er liegt im Bereich ihrer Art. 5 und 6, welche die persönliche Freiheit, Dauer der Untersuchungshaft und andere Aspekte des Strafverfahrens betreffen.

Knud Krakau

\title{
Alan Milner
}

\section{The Nigerian Penal System}

London: Sweet \& Maxwell. 1972. (Law in Africa. Number 32) $\$ 4.50$

Afrikanischer Strafvollzug wurde erst kürzlich wieder durch die spektakuläre Aktion des Präsidenten Bokassa der Zentralafrikanischen Republik, der durch Angehörige der Armee körperliche Strafen an Dieben mit teilweise tödlichem Erfolg vollziehen ließ, ins westliche Bewußtsein gebracht. Schlagartig wurde deutlich, wie unterschiedlich die gesellschaftliche Reaktion auf den Rechtsbruch in der westlichen Welt und Afrika ist. Was uns als eine grausame Negation menschlicher Grundrechte erscheint, ist für den Afrikaner sowohl der Form wie der der Handlung zugrunde liegenden Philosophie nach Ausdruck gewohnheitsrechtlicher und damit sozial anerkannter und akzeptierter Kriminalpolitik, deren plötzlicher Ausbruch vielleicht verwundert, der aber ziemlich bruchlos in die vom Gewohnheitsrecht entwickelten Sanktionen einzuordnen ist.

Auch Nigeria kennt für viele Straftaten noch die Prügelstrafe. So erlaubt der Indian Hemp Decree die Verhängung von 49 Stockschlägen, auszuführen an 
sieben Tagen, der Fire Arms and Robbery Decree erlaubt 24 Schläge, und die moderne Gesetzgebung im Norden des Landes hat die untere Grenze für die Zahl von Schlägen für zahlreiche Delikte auf 12 festgesetzt.

Wen die Konfliktsituation interessiert, die sich aus der Verdrängung des strafrechtlichen Gewohnheitsrechts in afrikanischen Ländern und der Einführung westlich orientierter Strafgesetze, Strafideen und Strafvollzugsinstitutionen ergibt, findet dazu viel Material und manche Gedanken in dem Buch von Alan Milner über das Strafen und den Strafvollzug in Nigeria. Der Autor hat selbst mehrere Jahre in Zaria im Norden Nigerias als Dean der dortigen Law Faculty gelebt und in dieser Zeit hinreichend Gelegenheit gehabt, das vorgelegte Material zu sammeln und die Komplexität des sozialen Gefüges eines Landes wie Nigeria zu studieren, in dem die Unterschiede zwischen traditionellen Dörfern und modernisierten Städten, zwischen so gegensätzlichen Völkern wie den Yoruba, Haussa-Fulani und Ibos, zwischen Islam und Animismus, zwischen britisch oktroyierter und nigerianisch bestimmter, aber dennoch überwiegend englisch orientierter Kriminalpolitik zu vielfältigen Erscheinungsformen des Verbrechens, aber auch der die Verbrechen bekämpfenden Institutionen und Maßnahmen geführt hat.

Anhand von zugegebenermaßen lückenhaften und nicht immer zuverlässigen kriminal-statistischen Ziffern aus den verschiedenen Staaten Nigerias vor Ausbruch der Unruhen von 1966 und vor dem Biafra-Krieg 1967-1970, zeichnet der Autor ein Bild der nigerianischen Kriminalität, in dem nicht nur häufig bürgerkriegsbedingter Straßenraub, Brandstiftung, illegaler Schußwaffengebrauch vorkommen, sondern auch die mit Hexenwesen und Jujuzauber verbundenen alten Praktiken ebenso eine Rolle spielen wie die individuelle Selbsthilfe und die kriminellen Folgeerscheinungen urbaner Entwicklung.

Den in Kapitel 2 dargestellten Sanktionen des Gewohnheitsstrafrechts stellt er die Probleme moderner Strafensysteme gegenüber, die mit der Übernahme britischer Strafgesetze und Strafarten - sei es direkt, sei es auf dem Umweg über Pakistan und den Sudan - auch in Nigeria akut geworden sind. Im Rahmen der Erörterung kriminalpolitischer Ziele beschäftigt er sich eingehend mit den Strafzwecken und den richterlichen Strafentscheidungen, die durch Einführung von festen Strafen - Todesstrafe für qualifizierten Raub oder Gefängnisstrafe ohne die Alternative der Geldstrafe bei leichter Kriminalität, hohe Maximumstrafen von nicht unter 21 Jahren Gefängnis oder Minimumbegrenzungen von 14, 12 und 10 Jahren für Rauschgiftdelikte, Staatsschutzdelikte und andere räuberische Handlungen - von der Militärregierung stark eingeschränkt wurden. Milner bleibt aber nie in der Theorie haften - und so prüft er die Anwendung der vom Gesetzgeber angedrohten Strafen in der Praxis. Dabei stellt sich heraus, daß die Richter der Native Courts eine Abneigung gegen die Verhängung von Freiheitsstrafen haben und häufiger Geldstrafen verhängen als die mit Berufsrichtern besetzten Gerichte. Es kann also der Schluß gezogen werden, daß die Auswahl der Strafart zumindest oberflächlich durch die kulturelle Herkunft des Richters beeinflußt wird. Hier zeigt sich zudem, wie hergebrachte, rechtliche Gebräuche den Einfluß eines neuen, fremden Systems überleben und immer wieder durchbrechen.

Weiten Raum widmet Milner sodann den herkömmlichen schlichtenden Verfahren des afrikanischen Rechts: Entschädigung, Rückgabe des durch die Straftat Erlangten und Beilegung des Streits durch Diskussion zwischen den beteiligten Parteien. Diese Maßnahmen haben auch in die Gesetze Eingang gefunden und werden neben 
den klassischen westlichen Strafen wie Geldstrafe, Freiheitsentzug und Todesstrafe häufig angewendet.

Kapitel 8, 9 und 10 sind der Freiheitsstrafe und ihrem Vollzug gewidmet. Obwohl die erzieherische und ausbildende Funktion des Gefängnisses den zuständigen Behörden Nigerias theoretisch durchaus bekannt ist, setzt doch die Praxis jeder konstruktiven Anleitung und Arbeit in den einzelnen Anstalten häufig enge Grenzen. Der jährliche Gefängnisbericht des Prison Department berichtet zwar über Tätigkeiten der Gefangenen wie Nähen, Weben, Bauen, Zigelherstellung, Schmieden, Sticken, Schustern, Geflügelhaltung, Gärtnern etc., doch scheitert eine sinnvolle und erfolgreiche Ausbildung der zumeist ungelernten Strafgefangenen häufig am Mangel an Ausbildern, an Ausbildungsplätzen, am Interesse und der Eignung des Gefangenen selbst sowie an fehlenden Arbeitsplätzen nach der Entlassung. Außer handwerklicher Ausbildung werden in manchen Gefängnissen Schreib- und Leselernkurse, aber auch die Vorbereitungsmöglichkeit für qualifiziertere Tätigkeiten angeboten. Besonders erfolgreich ist in dieser Hinsicht das Kakuri-PrisonCamp, ein Gefängnis für Langzeit-Inhaftierte. Im Ergebnis ist Milner über die positiven Erfolge des Freiheitsentzuges recht pessimistisch. Rigorose Gefängnisstrafen sind seiner Meinung nach auch in Afrika kein Weg, um die Kriminalität zu vermindern, auch führt ein langer Aufenthalt im Gefängnis - sei es in Nigeria oder sonstwo - nicht zu einer Resozialisierung des Betroffenen. Noch fragwürdiger ist die Koppelung langjähriger Freiheitsstrafen mit körperlichen Strafen, die auch in Nigeria nicht ungern verhängt werden. Über die mangelnde abschreckende Wirkung der Todesstrafe ist überall auf der Welt diskutiert worden. Die kriminalstatistisch unzuverlässigen Erhebungen Nigerias tragen wenig dazu bei, die allgemeine Diskussion zu befruchten. In Nigeria wird die Todesstrafe für zahlreiche Strafarten angedroht, vollstreckt jedoch meist nur in Mordfällen.

In dem letzten Kapitel seines Buches versucht der Autor, Vorschläge zur zukünftigen Kriminalpolitik des Landes zu machen. Er hat diese Ideen bereits 1970 auf einer internationalen Tagung für Kriminologie, die sich mit der nicht institutionellen Behandlung von Straftätern befaßte, einem größtenteils nigerianischen Publikum in Lagos vorgetragen. Er spart dabei nicht mit Kritik an den nigerianischen Verhältnissen. Die hohe Zahl der Gefängnisinsassen sowie die unzureichenden Personal- und Sachmittel machen jede konstruktive Erziehung und Rehabilitation zu einer fast unlösbaren Aufgabe. Aus Platzgründen können hier nicht alle Vorschläge Milners angeführt werden. Erwähnt sei nur seine Mahnung an die Richter, die bisher eine vom Volk getrennte Elite bilden, sich stärker über die Auswahl von Strafen, über deren Ziele und den Strafvollzug zu unterrichten. An die Regierung wendet sich der Autor mit der Bitte, Mittel zur Erforschung der Wirksamkeit von Strafen zur Verfügung zu stellen. Außerdem rät er der Regierung, die für die einzelnen Straftaten angedrohten Strafen neu zu durchdenken. In diesem Prozeß solle besonders darauf geachtet werden, welche traditionellen Maßnahmen ihren Zweck noch erfüllen können. Die Vorschläge Milners sind das konkrete Ergebnis einer jahrelangen Beschäftigung mit dem nigerianischen Strafrecht und können für die Regierung eine hilfreiche Grundlage für jede Reform auf dem Gebiet des Strafens und des Strafvollzuges werden.

Das Buch zeugt von gründlicher Kenntnis des behandelten Materials und der nicht immer nur nigerianischen Probleme. Es ist vom Thema, dem Stil und der Zuverlässigkeit her nicht nur für nigerianische Studenten und Praktiker von größtem 
Wert, sondern auch für den europäischen Juristen von nicht zu leugnender Aktualität und faszinierender Eindringlichkeit.

Barbara Huber

David E. Mutchler

The Church as a Political Factor in Latin America. With Particular Reference to Colombia and Chile

New York, Washington, London (Praeger Publishers) 1971. 460 Seiten.

Frederick C. Turner

Catholicism and Political Development in Latin America

Chapel Hill (The University of North Carolina Press) 1971. 272 Seiten.

Die Diskussion über die politische Rolle der Kirche in Lateinamerika hat praktisch nicht mehr abgerissen, seit die kritische Geschichtswissenschaft die Funktion des Katholizismus innerhalb des kolonialen Ausbeutungs- und Unterdrückungssystems u. a. auch im Sinne einer durchsichtigen, säkularen Herrschaftsideologie ausgeleuchtet hat. Obwohl die Kirche in Lateinamerika seither immer eine eminent politische, nämlich systemstabilisierende Rolle gespielt hat, kommt erst jetzt diese Diskussion in eine breitere Offentlichkeit, und zwar augenfällig im Zusammenhang mit sehr kritischen und progressiven Tendenzen innerhalb der Kirche selbst, gegen die von seiten der systemstabilisierenden Kräfte die politische Enthaltsamkeit gepredigt wird; daß letztere solche Enthaltsamkeit weder selbst beherzigen noch in der Vergangenheit beherzigt haben, wird dabei tunlichst verschwiegen, wobei ihnen der optische Tatbestand zugute kommt, daß sich politische Opposition naturgemäß anders artikulieren muß als die Kreise, die der politischen Herrschaft nahestehen.

Mutchler - "The Church as a Political Factor in Latin America“ ist ein gelungener Versuch, im Detail den politischen Zusammenhang aufzudecken, in dem die Kirche sich gegenwärtig in Lateinamerika bewegt. Mutchler war zwischen 1959 und 1967 selber Angehöriger der Societas Jesu und hat sich seither als ein hervorragender Kenner des lateinamerikanischen Katholizismus erwiesen; zeitweilig gehörte er der Facultad Latinoamericana de Ciencias Sociales (FLACSO) an; er hat an der Washington University von St. Louis in Philosophie mit einer Dissertation promoviert, die Grundlage für die vorliegende Arbeit war.

Mutchler beschränkt sich im wesentlichen auf die Rolle der Kirche in Columbien und Chile. Sein Hauptinteresse gilt ihrer organisatorischen Anpassung im Hinblick auf ihr Bestreben, Einfluß und Macht zu erhalten bzw. auszubauen. Seine Fragestellung weicht insofern in ihren Implikationen bereits von einer Vielzahl anderer religionssoziologischer Arbeiten der Weberschen Ausrichtung ab und orientiert sich mehr an der marxistischen Grundfrage nach latenten und manifesten Interessen auf dem Hintergrund bestimmter sozio-ökonomischer Lagen. Im einzelnen gehen dabei folgende drei Dimensionen in seine Analyse ein: erstens die gemeinsamen Interessen der lateinamerikanischen Kirchen, zweitens ihre materielle Basis und drittens ihr effektiver Entwicklungsbeitrag in den einzelnen Ländern. Seine gutrecherchierte Arbeit vermittelt sehr interessante Einblicke in die Organisation und die Aktivität der lateinamerikanischen Kirche auch und gerade im Hinblick auf ihre externen Verflechtungen und kommt zu sehr kritischen Schlußfolgerungen, die trotz der großen Publizität einiger sozialengagierter Gruppen daran zweifeln 